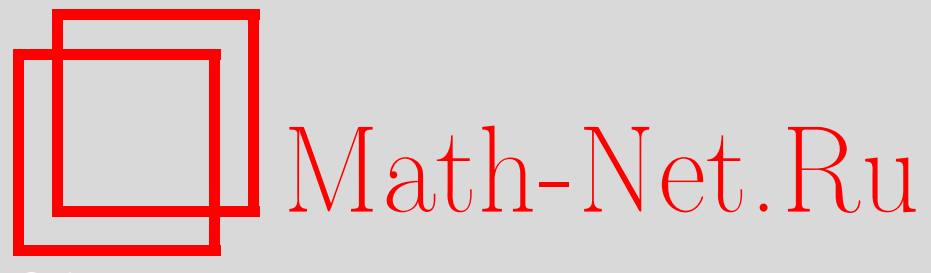

А. Д. Колесник, Э. Орсингер, Анализ двумерного случайного блуждания с конечной скоростью и отражением, Теория вероятн. и ее примен., 2001, том 46, выпуск 1, 138-147

DOI: https://doi.org/10.4213/tvp4016

Использование Общероссийского математического портала MathNet.Ru подразумевает, что вы прочитали и согласны с пользовательским соглашением

http://www . mathnet.ru/rus/agreement

Параметры загрузки:

IP : 54.224 .60 .19

26 апреля 2023 г., 18:20:38

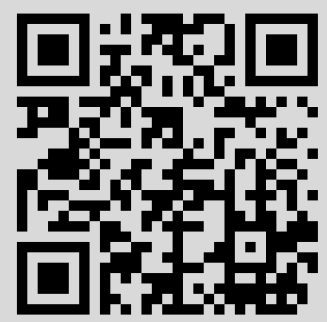


6. Нагаев С. В. Некоторые уточнения вероятностных и моментных неравенств. Теория вероятн. и ее примен., 1997, т. 42, в. 4, с. 832-838.

7. Nagaev $S . V$. On a new approach to the study of the distribution of a norm of a random element in Hilbert space. - Proceeding of the Fifth Vilnius Conference on Probability Theory and Mathematical Statistics. Vilnius: Mokslas, 1990, p. 214-226.

8. Johnson W. B., Schechtman G., Zinn J. Best constants in moment inequalities for linear combinations of independent and exchangeable random variables. - Ann. Probab., 1985, v. 13, p. 234-253.

9. Ибрагимов Р., ШІарахметов III. О точной константе в неравенстве Розенталя. Тезисы 4-го Ферганского международного коллоквиума по теории вероятностей и математической статистике. Ташкент, 1995, с. 43-44.

10. Ибрагимов Р., Шарахметов ШI. О точной константе в неравенстве Розенталя. Теория вероятн. и ее примен., 1997, т. 42, в. 2, с. 341-350.

11. Ибрагимов $P$., Шарахметов $\amalg$. Некоторые экстремальные проблемы в моментных неравенствах. - Теория вероятн. и ее примен. (в печати).

12. Прохоров Ю.В. Экстремальные задачи в предельных теоремах. - Труды VI Всесоюзного совещания по теории вероятностей и математической статистике. Вильнюс, 1962, с. $77-84$.

13. Пинелис И. $\Phi .$, Утев С. А. Оценки моментов сумм независимых случайных величин. - Теория вероятн. и ее примен., 1984, т. 29, в. 3, с. 554-557.

14. Утев C. A. Экстремальные задачи в моментных неравенствах. Труды Ин-та матем. СО АН СССР, 1985 , т. 5 , c. $56-75$.

15. Пешкир $\Gamma$., Ширяев $A$. H. Неравенства Хинчина и мартингальное расширение сферы их действия. - Успехи матем. наук, 1995, т. 50, в. 5, с. 3-62.

16. Ibragimov $R$., Sharakhmetov Sh. Exact bounds on the moments of symmetric statistics. - 7th Vilnius Conference on Probability Theory and Mathematical Statistics, 22nd European Meeting of Statisticians: Abstracts of Communications. Vilnius, 1998, p. 243-244.

17. Сачков В.Н. Комбинаторные методы дискретной математики. М.: Наука, 1977.

Поступила в редакцию 30.III.1998

Исправленный вариант 15.III.1999

(C) $2001 \mathrm{r}$.

КОЛЕСНИК А.Д.*, ОРСИНГЕР Э.**

\title{
АНАЛИЗ ДВУМЕРНОГО СЛУЧАЙНОГО БЛУЖДАНИЯ С КОНЕЧНОЙ СКОРОСТЬЮ И ОТРАЖЕНИЕМ ${ }^{1)}$
}

\begin{abstract}
Рассматривается двумерное случайное блуждание с конечной скоростью по четырем ортогональным направлениям и с возможностью отражения в моменты наступления пуассоновских событий. Мы получаем уравнение для распределения этого блуждания внутри области диффузии $Q_{t}$ и уравнения для его сингулярной компоненты. Распределения на границе области $Q_{t}$ и ее диагоналях получены в явном виде.
\end{abstract}

* Институт математики АН Молдовы, ул. Академическая, 5, 277028 Кишинев, Молдова; e-mail: kolesnik@math.md

** Dipartimento di Statistica, Probabilità e Statistiche Applicate, Università «La Sapienza», P. le Aldo Moro 5, 00185 Roma, Italia.

1) Первый автор поддержан исследовательским грантом в рамках NATO-CNR Guest Fellowships Programme. 
Ключевые слова и фразы: двумерное случайное блуждание, конечная скорость, функции Бесселя, гиперболические уравнения, телеграфное уравнение.

1. Введение. В настоящей работе мы рассматриваем случайное блуждание на плоскости с конечной скоростью $c$ и четырьмя возможными направлениями движения. Такого рода блуждания, управляемые однородным процессом Пуассона с параметром $\lambda>0$, исследовались в ряде работ для разного числа направлений и различных способов их изменения (см. [5], [13], [12], [8], [1]). Распределения положения частиц, движущихся вышеописанным образом, являются решениями гиперболических уравнений, порядок которых совпадает с числом возможных направлений. Это влечет существенное усложнение вероятностного анализа таких моделей. По нашему мнению, именно это обстоятельство объясняет, почему одномерные блуждания с конечной скоростью интенсивно исследовались во всех деталях (см. [9], [11], [2], [3], [14], [6]), тогда ках их многомерные аналоги почти полностью игнорировались.

Для того чтобы вывести требуемые уравнения и, таким образом, получить информацию о распределении случайным образом движущейся частицы, мы предполагаем, что разрешенные направления движения подчинены соответствующим условиям симметрии. Несмотря на то, что блуждание по трем направлениям описывается уравнением третьего порядка (и это есть минимальное число направлений, при котором получается нетривиальная модель на плоскости), нам кажется, что блуждание с четырьмя взаимно ортогональными направлениями, описываемое гиперболическим уравнением четвертого порядка, является разумным компромиссом между математической сложностью и реалистическим представлением модели.

Двумерные блуждания такого рода рассматривались в некоторых предшествующих работах. В частности, В [12] мы предполагали, что после каждого изменения направления частица может двигаться только по направлению, ортогональному предыдущему (влево или вправо), и что граница диффузионной области $Q_{t}$ достижима только при одном изменении направления. Это последнее, довольно искусственное, условие было исключено в статье [10], где граница могла достигаться частицей при любом числе изменений направления ее движения.

В настоящей работе мы предполагаем, что при наступлении каждого пуассоновского события частица может менять направление своего движения либо на одно из двух ортогональных, либо на обратное (т.е. она может отражаться назад, как шар на бильярдном столе). Это существенным образом меняет математическое описание движения со многих точек зрения. Если в модели, исследованной авторами в [12], волновой Фронт, распространяющийся со скоростью $c$, представляет собой единое множество, на котором сосредоточена сингулярная компонента распределения, то в анализируемом здесь случае диагонали области $Q_{t}$ также содержат часть сингулярной компоненты распределения. Это происходит потому, что вначале частица двигается вдоль осей $x$ и $y$ (в обоих направлениях), и при наступлении каждого пуассоновского события она может двигаться по той же самой прямой в обратном направлении. Другими словами, она может осуществлять одномерное движение по одной из координатных осей с положительной вероятностью. Кроме того, частица может достигать гранищы $\partial Q_{t}$ диффузионной области $Q_{t}$ при любом числе изменений направления движения. Если изменений направления не происходит, то частица достигает одной из вершин области $Q_{t}$.

Допущение о возможности отражения влечет значительные отличия от блуждания, в котором траектории движения состоят только из взаимно ортогональных сегментов. В рассматриваемой здесь модели, в силу возможности отражения при каждом изменении направления, частица может с положительной вероятностью оставаться на некоторой прямой, затем она может совершать одномерное движение на интервале времени между некоторыми двумя непоследовательными пуассоновскими событиями и затем вернуться (или нет) к двумерному движекию. В терминах траекторий это означает, что мера траекторий, имеющих ненулевую меру самопересечений, положительна.

Рассмотренное здесь случайное движение представляется хорошей моделью для конкретных блужданий таких, как, к примеру, движение электрических зарядов в 
плоских проводниках, где изменения направлений движения вызываются столкновениями зарядов с молекулами вещества, или случайные блуждания, появляющиеся в некоторых компьютерных играх. В реальных случайных блужданиях важную роль играет инерция, так что траектории случайным образом движущихся частиц состоят из сегментов, как и в модели, рассмотренной ниже.

Заметим, что если скорость $c \rightarrow \infty$ и интенсивность переключающего пуассоновского процесса $\lambda \rightarrow \infty$ таким образом, что $c^{2} / \lambda \rightarrow 1$, то уравнение для распределения случайного блуждания превращается в двумерное уравнение теплопроводности. Более того, в этом случае частица начинает совершать двумерное броуновское движение с непрерывным изменением направлений и бесконечной скоростью распространения. Это позволяет нам интерпретировать нашу модель как конечно-скоростную аппроксимацию двумерного броуновского движения.

2. Описание модели и структура распределения. Частица стартует в момент времени $t=0$ из начала координат $x=y=0$ плоскости $\mathbf{R}^{2}$ и движется с постоянной конечной скоростью $c$. В каждый момент времени частица может иметь одно из четырех возможных направлений движения $E_{k}=(\pi k / 2), k=0,1,2,3$, причем выбор начального направления делается с равными вероятностями $\frac{1}{4}$. Это означает, что направление движения частищы $D(t)$ есть случайный процесс с четырьмя состояниями $E_{k}, k=0,1,2,3$. Изменения направлений управляются однородным процессом Пуассона с параметром $\lambda>0$ следующим образом. При каждом наступлении пуассоновского события частица мгновенно выбирает одно из остальных направлений с равными вероятностями $\frac{1}{3}$ и продолжает движение в выбранном направлении до наступления следующего пуассоновского события, затем опять выбирает новое направление описанным выше способом и т.д.

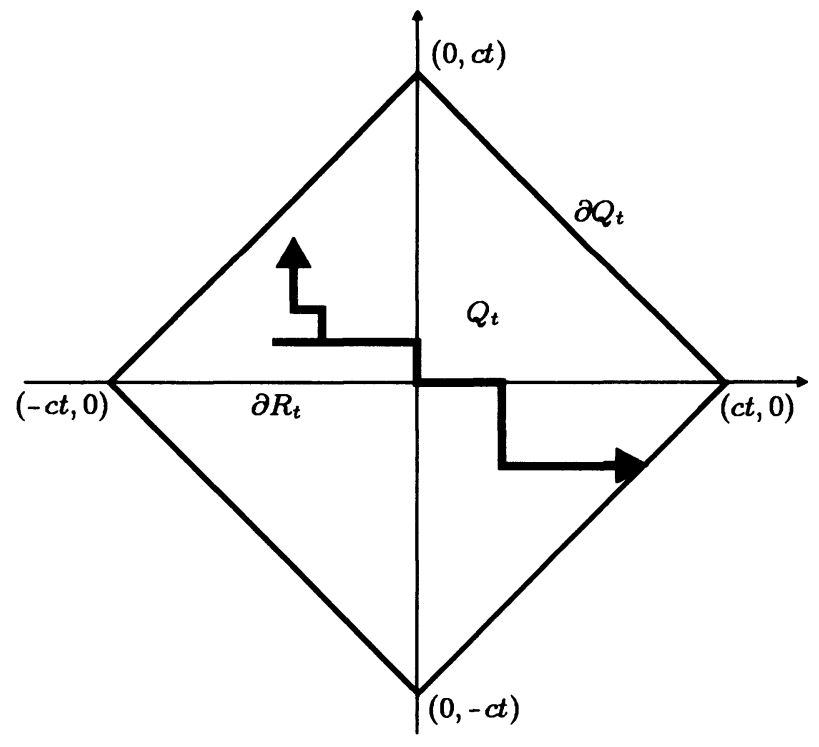

Рис. 1. Пример двух траекторий при 2 и 5 пуассоновских событиях.

Отметим, что здесь мы предполагаем, что при каждом наступлении пуассоновского события частица может двигаться в обратном направлении (с вероятностью $\frac{1}{3}$ ), и это значительно отличает настоящую модель от рассмотренной авторами в [12]. Этот эффект отражения меняет нашу модель самым существенным образом.

Пусть $(X(t), Y(t))$ обозначает положение частицы в момент времени $t$. Целью нашего исследования является анализ этого процесса и его основных свойств.

Для того чтобы описать структуру распределения процесса $(X(t), Y(t))$, мы заметим, что оно имеет абсолютно непрерывную компоненту, поскольку траектории 
процесса непрерывны и регулярны (т.е. дифференцируемы почти всюду), и скорость распространения конечна.

$\mathrm{B}$ произвольный момент времени $t>0$ частица с вероятностью 1 находится в косом квадрате

$$
Q_{t}=\left\{(x, y) \in \mathbf{R}^{2}:|x+y| \leqslant c t,|x-y| \leqslant c t\right\}
$$

(см. рис. 1), представляющем собой носитель распределения.

Поскольку в любой момент времени $t>0$ частица может с положительной вероятностью находиться как на внешней границе

$$
\partial Q_{t}=\left\{(x, y) \in Q_{t}:|x \pm y|=c t\right\}
$$

квадрата $Q_{t}$ (включая углы), так и на осях (диагоналях $\left.Q_{t}\right)$, т.е. множестве

$$
\partial R_{t}=\left\{(x, y) \in Q_{t}: x=0 \vee y=0\right\}
$$

то сингулярная компонента распределения состоит из двух частей, одна из которых относится к множеству $\partial Q_{t}$, а другая к множеству $\partial R_{t}$. Это дает меру всех траекторий процесса, начинающихся в момент времени $t=0$ в начале координат $x=y=0$ и заканчивающихся в момент $t$ либо на внешней границе $\partial Q_{t}$, либо на диагоналях $\partial R_{t}$.

Заметим, что частица в момент $t$ может с положительной вероятностью находиться на оси, первоначально выбранной ею, и это есть вероятность того, что за время $t$ при наступлении пуассоновских событий частица ни разу не покинула диагональ $Q_{t}$. Очевидно, что если до момента времени $t$ произошло $k, k \geqslant 1$, пуассоновских событий, то вероятность того, что частица находится на одной из осей $\partial R_{t}$ равна $1 / 3^{k}$.

Следовательно, вероятность того, что в момент времени $t$ частица находится на одной из осей $\partial R_{t}$, есть

$$
\mathbf{P}\left\{(X(t), Y(t)) \in \partial R_{t}\right\}=\sum_{k=1}^{\infty} \frac{1}{3^{k}} \mathbf{P}\{N(t)=k\}=e^{-2 \lambda t / 3}-e^{-\lambda t},
$$

где $N(t)$ обозначает число пуассоновских событий, происшедших до момента $t$.

Если до момента времени $t$ произошло $k, k \geqslant 1$, пуассоновских событий, то частица будет находиться на внешней границе $\partial Q_{t}$ с вероятностью $\frac{2}{3} \cdot\left(1 / 3^{k-1}\right)$. Это означает, что вероятность нахождения частицы в момент $t$ на внешней границе $\partial Q_{t}$ (включая углы) равна

$$
\mathbf{P}\left\{(X(t), Y(t)) \in \partial Q_{t}\right\}=e^{-\lambda t}+\frac{2}{3} \sum_{k=1}^{\infty} \frac{1}{3^{k-1}} \mathbf{P}\{N(t)=k\}=2 e^{-2 \lambda t / 3}-e^{-\lambda t}
$$

Оставшаяся компонента распределения абсолютно непрерывна и сосредоточена в области $Q_{t}-\partial Q_{t}-\partial R_{t}$. Сравнивая (2.4) и (2.5), можно заключить, что вероятность нахождения на $\partial Q_{t}$ (с углами или без) всегда больше вероятности нахождения на $\partial R_{t}$.

Как мы отмечали выше, поскольку при каждом изменении направления частица может отражаться назад, то с вероятностью (2.4) она не покинет выбранную диагональ $\partial R_{t}$ на интервале времени $[0, t)$. В этом случае она совершает чисто одномерное движение, которое мы опишем в следующем пункте.

3. Описание движения на осях $\partial R_{t}$ и его точное распределение. Пусть частица движется на одной из осей $\partial R_{t}$, скажем, $O X$. Если обозначить через $\zeta(t)$ случайное направление на оси $O X$ в момент времени $t$, принимающее значения + или при движении в положительном или отрицательном направлениях соответственно, то можно рассмотреть следующие совместные плотности:

$$
\begin{aligned}
& f(x, t) d x=\mathbf{P}\{x \leqslant X(t)<x+d x, \zeta(t)=+\} \\
& g(x, t) d x=\mathbf{P}\{x \leqslant X(t)<x+d x, \zeta(t)=-\}
\end{aligned}
$$


Для этих плотностей можно выписать уравнение Колмогорова, которое в нашем случае представляет собой гиперболическую систему двух уравнений в частных производных с постоянными коэффициентами

$$
\frac{\partial f}{\partial t}=-c \frac{\partial f}{\partial x}-\lambda f+\frac{\lambda}{3} g, \quad \frac{\partial g}{\partial t}=c \frac{\partial g}{\partial x}-\lambda g+\frac{\lambda}{3} f .
$$

Для вывода уравнений (3.1) следует учесть, что при каждом пуассоновском событии частица может оставаться на оси $O X$ с вероятностью $\frac{1}{3}$.

Для нас особенно важна функция $p(x, t)=f(x, t)+g(x, t)$, представляющая собой плотность положения частицы на оси $O X$.

Дифференцирован ием уравнений системы (3.1) и соответствующими подстановками (либо простым вычислением формального определителя системы (3.1), см. [7]) можно получить уравнение для плотности $p=p(x, t)$ :

$$
\frac{\partial^{2} p}{\partial t^{2}}+2 \lambda \frac{\partial p}{\partial t}-c^{2} \frac{\partial^{2} p}{\partial x^{2}}+\frac{8 \lambda^{2}}{9} p=0
$$

Наш первый результат касается явного вида функции $p=p(x, t)$.

Теорема 1. Плотность $p=p(x, t), x \in \mathbf{R}^{1}, t>0$, распределения положения частичы на оси $O X$ имеет следующиนи вид:

$$
p(x, t)=\frac{e^{-\lambda t}}{4 c}\left[\frac{\lambda}{3} I_{0}\left(\frac{\lambda}{3 c} \sqrt{c^{2} t^{2}-x^{2}}\right)+\frac{\partial}{\partial t} I_{0}\left(\frac{\lambda}{3 c} \sqrt{c^{2} t^{2}-x^{2}}\right)\right],
$$

где $|x|<$ сt и $I_{0}(x)$ есть фуккиия Бесселя мнимого аргумента нулевого рода.

Д о к а $з$ а т е л ь с т в о. С помощью замены $p(x, t)=e^{-\lambda t} w(x, t)$ уравнение (3.2) преобразуется к виду

$$
\frac{\partial^{2} w}{\partial t^{2}}-c^{2} \frac{\partial^{2} w}{\partial x^{2}}-\frac{\lambda^{2}}{9} w=0
$$

Известно (см. например, [9]), что функция

$$
w(x, t)=I_{0}\left(\frac{\lambda}{3 c} \sqrt{c^{2} t^{2}-x^{2}}\right)
$$

для $|x|<$ ct является неотрицательным решением (3.4) (как и ее производные по времени). Следовательно, в соответстви и с (2.4), нам нужно лишь проверить, что

$$
\int_{-c t}^{c t} p(x, t) d x=\frac{1}{2}\left(e^{-2 \lambda t / 3}-e^{-\lambda t}\right) .
$$

Учитывая, что (см. [7])

$$
\begin{aligned}
\int_{-c t}^{c t} I_{0}\left(\frac{\lambda}{3 c} \sqrt{c^{2} t^{2}-x^{2}}\right) d x & =\frac{3 c}{\lambda}\left(e^{\lambda t / 3}-e^{-\lambda t / 3}\right), \\
\int_{-c t}^{c t} \frac{\partial}{\partial t} I_{0}\left(\frac{\lambda}{3 c} \sqrt{c^{2} t^{2}-x^{2}}\right) d x & =c\left(e^{\lambda t / 3}+e^{-\lambda t / 3}\right)-2 c,
\end{aligned}
$$

мы немедленно получаем (3.6).

3 а м е ч а н и е. Распределение на оси $O Y$ получается автоматически из (3.3) формальной заменой $x$ на $y$.

4. Точное распределение на внешней границе $\partial Q_{t}$. После первоначального одномерного движения по координатной оси частица начинает совершать чисто двумерное блуждание, когда после наступления некоторого пуассоновского события она меняет свое направление на одно из ортогональных и покидает координатную ось (с вероятностью $\frac{2}{3}$ ). Уравнение для распределения этого случайного блуждания будет дано в следующем пункте. Здесь же мы получим явный вид распределения положения частицы на внешней границе $\partial Q_{t}$.

Очень важно сразу заметить, что частица будет находиться на границе $\partial Q_{t}$ в момент времени $t$, если и только если она находилась на границе $\partial Q_{t}$ в течение всего 
интервала времени $[0, t)$, ни разу не покинув ее. Это вытекает из того факта, что область диффузии $Q_{t}$ распространяется вовне, и каждая точка границы $\partial Q_{t}$ двигается вовне с векторной скоростью $\mathbf{v}=(c / \sqrt{2})(\mathbf{i}+\mathbf{j})$, где $\mathbf{i}, \mathbf{j}$ суть ортонормальные векторы. Это означает, что, раз покинув границу, частица никогда не сможет на нее вернуться. Следовательно, каждая точка границы $\partial Q_{t}$ в момент времени $t$ является концевой точкой некоторой траектории. Для того чтобы частица двигалась вместе с границей, она должна при каждом пуассоновском событии выбирать только направление вовне (это происходит с вероятностью $\frac{1}{3}$ ).

Нашей целью здесь является нахождение точного распределения положения частицы на границе $\partial Q_{t}$ в произвольный момент времени $t>0$. Симметричная структура модели позволяет нам, вместо переменных $x$ и $y$ в отдельности, использовать их сумму и разность

$$
\xi=x+y, \quad \eta=x-y .
$$

Мы рассмотрим распределение положения частицы на стороне $\partial Q_{t}^{1}$ гранищы $\partial Q_{t}$, расположенной в первом квадранте (т.е. на прямой $x+y=\xi=c t$ ). Очевидно, для того чтобы частица находилась на $\partial Q_{t}^{1}$ в момент времени $t$, она должна была выбрать начальное направление $E_{0}$ либо $E_{1}$, а затем чередовать только эти два направления. Важно подчеркнуть, что положение частицы на $\partial Q_{t}^{1}$ однозначно определяется второй координатой $\eta$.

Введем следующие совместные плотности:

$$
\begin{aligned}
& f_{0}(\eta, t) d \eta=\mathbf{P}\left\{(X(t), Y(t)) \in \partial Q_{t}^{1}, \quad \eta \leqslant(X(t), Y(t))<\eta+d \eta, D(t)=E_{0}\right\}, \\
& f_{1}(\eta, t) d \eta=\mathbf{P}\left\{(X(t), Y(t)) \in \partial Q_{t}^{1}, \quad \eta \leqslant(X(t), Y(t))<\eta+d \eta, D(t)=E_{1}\right\} .
\end{aligned}
$$

Простые рассуждения показывают, что функции (4.2) удовлетворяют гиперболической системе

$$
\frac{\partial f_{0}}{\partial t}=-c \frac{\partial f_{0}}{\partial \eta}-\lambda f_{0}+\frac{\lambda}{3} f_{1}, \quad \frac{\partial f_{1}}{\partial t}=c \frac{\partial f_{1}}{\partial \eta}-\lambda f_{1}+\frac{\lambda}{3} f_{0} .
$$

Функция $p=f_{0}+f_{1}$ представляет собой плотность вероятности положения частицы на $\partial Q_{t}^{1}$, удовлетворяющую гиперболическому уравнению второго порядка:

$$
\frac{\partial^{2} p}{\partial t^{2}}+2 \lambda \frac{\partial p}{\partial t}-c^{2} \frac{\partial^{2} p}{\partial \eta^{2}}+\frac{8 \lambda^{2}}{9} p=0
$$

Приведенные выше рассуждения применимы и к остальным сторонам квадрата $\partial Q_{t}$ (т.е. для $\left.\partial Q_{t}^{i}, i=2,3,4\right)$. В частности, уравнение $(4.4)$, также справедливо для плотности, сосредоточенной на $\partial Q_{t}^{3}$, а распределение на остальных двух сторонах удовлетворяет уравнению, аналогичному (4.4), с формальной заменой $\eta$ на $\xi$.

Основной результат этого пункта дается следующей теоремой.

Теорема 2. Для всех $(x, y) \in \partial Q_{t}$ (без углов) и любого $t>0$ распределение ка граяиче имеет следующий вид:

$$
p(x, y, t)=\left\{\begin{aligned}
\frac{e^{-\lambda t}}{2 c} & {\left[\frac{\lambda}{3} I_{0}\left(\frac{\lambda}{3 c} \sqrt{c^{2} t^{2}-(x+y)^{2}}\right)\right.} \\
& \left.+\frac{\partial}{\partial t} I_{0}\left(\frac{\lambda}{3 c} \sqrt{c^{2} t^{2}-(x+y)^{2}}\right)\right] \delta(x-y \pm c t), \\
\frac{e^{-\lambda t}}{2 c} & {\left[\frac{\lambda}{3} I_{0}\left(\frac{\lambda}{3 c} \sqrt{c^{2} t^{2}-(x-y)^{2}}\right)\right.} \\
& \left.+\frac{\partial}{\partial t} I_{0}\left(\frac{\lambda}{3 c} \sqrt{c^{2} t^{2}-(x-y)^{2}}\right)\right] \delta(x+y \pm c t),
\end{aligned}\right.
$$

где $I_{0}$ есть функиия Бесселя мнимого аргумента кулевого рода и $\delta(\cdot)$ есть функчия Дираха. 
Д о к а з а т е л с с т в о. Проверка того, что (4.5) является неотрицательным решением уравнения (4.4), проводится аналогично тому, как это было сделано при доказательгтве теоремы 1.

Мы проверим сейчас, что (4.5) удовлетворяет условию

$$
\iint_{\partial Q_{t}-\mathbf{y г л \mathbf { ~ }}} p(x, y, t) d x d y=2 e^{-2 \lambda t / 3}-2 e^{-\lambda t} .
$$

В силу симметрии функции (4.5), достаточно убедиться (делая преобразование (4.1)), что

$$
\begin{aligned}
& \iint_{\partial Q_{t}^{1}-\text { углы }} p(x, y, t) d x d y=\frac{1}{2} \int \delta(\xi-c t) d \xi \\
& \quad \times \int_{-c t}^{c t} \frac{e^{-\lambda t}}{2 c}\left[\frac{\lambda}{3} I_{0}\left(\frac{\lambda}{3 c} \sqrt{c^{2} t^{2}-\eta^{2}}\right)+\frac{\partial}{\partial t} I_{0}\left(\frac{\lambda}{3 c} \sqrt{c^{2} t^{2}-\eta^{2}}\right)\right] d \eta \\
& =\frac{1}{2}\left(e^{-2 \lambda t / 3}-e^{-\lambda t}\right) .
\end{aligned}
$$

На последнем шаге мы воспользовались формулами (3.7) и (3.8). Теперь, чтобы получить условие (2.5), нужно лишь умножить последнее равенство на 4 и прибавить вероятность, сосредоточенную в вершинах квадрата $\partial Q_{t}\left(\right.$ т.е. $\left.e^{-\lambda t}\right)$.

5. Об абсолютно непрерывной компоненте распределения. Теперь мы займемся анализом блуждания в области $Q_{t}-\partial Q_{t}-\partial R_{t}$. Внутри этой области распределение абсолютно непрерывно, и, стало быть, можно говорить о его плотности.

Для каждого направления $E_{k}, k=0,1,2,3$, мы введем совместное распределение положения частицы и ее направления $f_{k}(x, y, t) d x d y=\mathbf{P}\{x \leqslant X(t)<x+d x, y \leqslant$ $\left.Y(t)<y+d y, D(t)=E_{k}\right\}, k=0,1,2,3$. Уравнение Колмогорова, записанное для функций $f_{k}=f_{k}(x, y, t),(x, y) \in Q_{t}-\partial Q_{t}-\partial R_{t}, t>0$, приводит к следуюшей гиперболической системе уравнений в частных производных первого порядка с постоянными коэффициентами:

$$
\frac{\partial f_{k}}{\partial t}=-c \cos \frac{\pi k}{2} \frac{\partial f_{k}}{\partial x}-c \sin \frac{\pi k}{2} \frac{\partial f_{k}}{\partial y}-\lambda f_{k}+\frac{\lambda}{3} \sum_{j=0, j \neq k}^{3} f_{j}, \quad k=0,1,2,3 .
$$

Для нас особый интерес представляет функция $p=p(x, y, t)$, определяемая как $p=f_{0}+f_{1}+f_{2}+f_{3}$, поскольку она есть не что иное, как плотность распределения положения частищы в области $Q_{t}-\partial Q_{t}-\partial R_{t}$.

С помощью довольно длинных, но простых вычислений, использующих последовательные дифференцирования, подстановки и исключения в системе (5.1), можно показать, что функция $p=p(x, y, t)$ (равно как и функции $f_{k}$ ) является решением гиперболического уравнения четвертого порядка с постоянными коэффициентами

$$
\begin{gathered}
\left(\frac{\partial^{4}}{\partial t^{4}}+4 \lambda \frac{\partial^{3}}{\partial t^{3}}+\frac{16 \lambda^{2}}{9} \frac{\partial^{2}}{\partial t^{2}}+\frac{64 \lambda^{3}}{27} \frac{\partial}{\partial t}-c^{2} \frac{\partial^{2}}{\partial t^{2}} \Delta-2 \lambda c^{2} \frac{\partial}{\partial t} \Delta\right. \\
\left.-\frac{8 \lambda^{2} c^{2}}{9} \Delta+c^{4} \frac{\partial^{4}}{\partial x^{2} \partial y^{2}}\right) p=0
\end{gathered}
$$

где $\Delta$ есть двумерный оператор Лапласа.

Заметим, что, в соответствии с [7], уравнение (5.2) может быть также получено из системы (5.1) вычислением ее формального определителя. Уравнение (5.2) является частным случаем (при $n=4$ ) более общего уравнения, описывающего двумерное случайное блуждание с $n, n \geqslant 2$, направлениями (см. [8]).

Отметим, что уравнение (5.2) при $c \rightarrow \infty, \lambda \rightarrow \infty, c^{2} / \lambda \rightarrow 1$, превращается в двумерное уравнение теплопроводности.

Уравнение (5.2) является гиперболическим уравнением с постоянными коэффициентами, и, как известно из общей теории уравнений в частных производных, для такого уравнения как задача Коши, так и краевая задача, ставятся корректными, причем гладкость решения полностью определяется гладкостью соответствующих начальных условий. Поскольку нам известно распределение на внешней границе $\partial Q_{t}$ 
и на осях $\partial R_{t}$ диффузионной области $Q_{t}$, то мы можем поставить краевую задачу с условиями на границе и на осях, задаваемыми функциями (4.5) и (3.3) соответственно. Помимо этого, можно также рассмотреть смешанную задачу для уравнения (5.2) с двумя нулевыми начальными условиями (поскольку в начальный момент абсолютно непрерывная компонента равна нулю, а интенсивность управляющего пуассоновского процесса постоянна во времени) и с остальными условиями на границе.

К сожалению обе эти задачи могут быть решены лишь численно, а аналитическое выражение для абсолютно непрерывной компоненты распределения, по-видимому, не может быть дано через известные функции. К такому выводу нас приводят следуюшие соображения.

Экспоненциальная подстановка

$$
p(x, y, t)=e^{-4 \lambda t / 3} w(x, y, t)
$$

приводит к более простому уравнению

$$
\left(\frac{\partial^{4}}{\partial t^{4}}-\frac{4 \lambda}{3} \frac{\partial^{3}}{\partial t^{3}}-c^{2} \frac{\partial^{2}}{\partial t^{2}} \Delta+\frac{2 \lambda c^{2}}{3} \frac{\partial}{\partial t} \Delta+c^{4} \frac{\partial^{4}}{\partial x^{2} \partial y^{2}}\right) w=0,
$$

так как все производные первого и второго порядков исчезают.

Преобразование Фурье $W(\alpha, \beta, t)=\iint_{\mathbf{R}^{2}} e^{i \alpha x+i \beta y} w(x, y, t) d x d y$ удовлетворяет обыкновенному дифференциальному уравнению

$$
\left\{\frac{d^{4}}{d t^{4}}-\frac{4 \lambda}{3} \frac{d^{3}}{d t^{3}}+c^{2}\left(\alpha^{2}+\beta^{2}\right) \frac{d^{2}}{d t^{2}}-\frac{2 \lambda c^{2}}{3}\left(\alpha^{2}+\beta^{2}\right) \frac{d}{d t}+c^{4} \alpha^{2} \beta^{2}\right\} W=0,
$$

корни характеристического уравнения которого были в явном виде вычислены с помощью пакета компьютерных программ МАТНЕМАТІСА и имеют крайне громоздкий вид. Это убеждает нас, что, по-видимому, общее решение уравнения (5.4) не может быть представлено в виде комбинации известных функций.

Однако, мы можем получить частные решения уравнения (5.2), имеющие форму, аналогичную распределениям на осях $\partial R_{t}$ и на внешней границе $\partial Q_{t}$ (см. теоремы 1 и 2). Кроме того, эти частные решения могут быть использованы для интегрирования уравнений (5.2) и (5.5).

Теорема 3. Для ^юбых $(x, y) \in Q_{t}$ и $t>0$ функииน

$$
\begin{aligned}
& q(x, y, t)=e^{-2 \lambda t / 3} I_{0}\left(\frac{2 \lambda}{3 c} \sqrt{c^{2} t^{2}-(x+y)^{2}}\right) \\
& r(x, y, t)=e^{-2 \lambda t / 3} I_{0}\left(\frac{2 \lambda}{3 c} \sqrt{c^{2} t^{2}-(x-y)^{2}}\right)
\end{aligned}
$$

являются решениями уравкения (5.2).

Д ок а за те ль с т в о. Как мы уже отмечали выше, экспоненциальное преобразование (5.3) сводит уравнение (5.2) к уравнению (5.4). Преобразованием вращения (4.1) уравнение (5.4) приводится к виду

$$
\left\{\left(\frac{\partial^{2}}{\partial t^{2}}-c^{2} \Delta\right)\left(\frac{\partial^{2}}{\partial t^{2}}-c^{2} \Delta-\frac{4 \lambda}{3} \frac{\partial}{\partial t}\right)-4 c^{4} \frac{\partial^{4}}{\partial \xi^{2} \partial \eta^{2}}\right\} \varphi(\xi, \eta, t)=0
$$

из которого немедленно вытекает утверждение теоремы, так как хорошо известно, что функции

$$
e^{2 \lambda t / 3} I_{0}\left(\frac{2 \lambda}{3 c} \sqrt{c^{2} t^{2}-\xi^{2}}\right), \quad e^{2 \lambda t / 3} I_{0}\left(\frac{2 \lambda}{3 c} \sqrt{c^{2} t^{2}-\eta^{2}}\right)
$$

являются решениями телеграфного оператора, появляющегося в (5.7).

3 а м е ч а н и е. Представляет интерес определение момента времени $t^{*}$, когда вес абсолютно непрерывной компоненты распределения равен весу его сингулярной компоненты, т.е. $\mathbf{P}\left\{(X(t), Y(t)) \in Q_{t}-\partial Q_{t}-\partial R_{t}\right\}=\mathbf{P}\left\{(X(t), Y(t)) \in \partial Q_{t} \cup \partial R_{t}\right\}$.

Для того чтобы определить $t^{*}$, в соответствии с (2.4) и (2.5), нужно решить уравнение

$$
6 e^{-2 \lambda t / 3}-4 e^{-\lambda t}-1=0
$$


Полагая $r=e^{-\lambda t / 3}$, мы получаем кубическое уравнение $4 r^{3}-6 r^{2}+1=0$ с корнями $r_{1}=\frac{1}{2}, r_{2}=\frac{1-\sqrt{3}}{2}, r_{3}=\frac{1+\sqrt{3}}{2}$. Легко видеть, что подходит только первый корень, дающий

$$
t^{*}=\frac{3}{\lambda} \ln 2
$$

Все это приводит к следующей картине поведения распределения. Первоначально большая часть вероятности сосредоточена на границе $\partial Q_{t}$ и на осях $\partial R_{t}$, и до момента времени $t^{*}$ эта компонента превалирует. После момента $t^{*}$ вес абсолютно непрерывной компоненты начинает преобладать, а сингулярная компонента все уменьшается с течением времени. Формула (5.8) показывает, что если интенсивность переключений $\lambda$ стремится к бесконечности, вес абсолютно непрерывной компоненты становится доминирующим мгновенно.

3 а м е ч н и е. Траектории, составляющие абсолютно непрерывную часть распределения, могут быть разбиты на два множества, одно из которых содержит траектории с нулевой мерой самопересечений (т.е. без отражения), а другое - с положительной мерой самопересечений (т.е. для которых частица отразилась хотя бы раз). Простые вычисления позволяют выписать вероятность того, что до момента времени $t$ не произошло ни одного отражения:

$$
\begin{aligned}
& \mathbf{P}\left\{\begin{array}{l}
\text { частица в момент } t \\
\text { находится внутри } Q_{t} \text { и } \\
\text { ни разу не отразилась }
\end{array}\right\}=\frac{1}{3} \mathbf{P}\{N(t)=2\}+\sum_{k=3}^{\infty}\left(\frac{2}{3}\right)^{k-3} \mathbf{P}\{N(t)=k\} \\
& =\frac{9}{4}\left[e^{-\lambda t / 3}-e^{-\lambda t}\left(1+\frac{2}{3} \lambda t+\frac{1}{3}\left(\frac{2}{3} \lambda t\right)^{2}\right)\right] .
\end{aligned}
$$

Оставшаяся часть абсолютно непрерывной компоненты распределения $\mathbf{P}\{X(t)<x$, $Y(t)<y\}$ относится к множеству траекторий с положительной мерой самопересечений.

A. Колесник благодарит Consiglio Nazionale delle Ricerche (CNR) Италии за поддержку этого исследования в рамках NATO-CNR Guest Fellowships Programme и отдел статистики и вероятности университета «La Sapienza» (Рим, Италия) за создание прекрасных рабочих условий. А. Колесник благодарен своему соавтору профессору Энцо Орсингеру за гостеприимство во время его пребывания в Риме. Авторы хотят поблагодарить д-ра Luisa Beghin, д-ра Stefano Iacus и д-ра Lila Di Caprio за их помощь в оформлении рукописи статьи. Авторы признательны рецензентам за содержательные замечания, позволившие внести улучшения в первоначальный вариант статьи.

\section{СПИСОК ЛИТЕРАТУРЫ}

1. Di Crescenzo A. Exact transient analysis of a planar random motion with three directions. Preprint No 13/99. Università della Basilicata, 1999.

2. Foong S.K. First-passage time, maximum displacement, and Kac's solution of the telegraph equation. - Phys. Rev., 1992, v. 46, p. 707-710.

3. Foong $S$. K., Kanno $S$. Properties of the telegrapher's random process with or without a trap. - Stochastic Process. Appl., 1994, v. 53, p. 147-173.

4. Кабаяов Ю. М. О вероятностном представлении решения телеграфного уравнения. - Теория вероятн. и ее примен., 1992, т. 37, в. 2, с. 425-426.

5. Колесних А.Д. Об одной модели марковской случайной эволюции на плоскости, Аналитические методы исследования эволюции стохастических систем. Киев: Ин-т матем. АН Украины, 1989, с. 55-61.

6. Kolesnik A.D. The equations of Markovian random evolution on the line. - J. Appl. Probab., 1998, v. 35, p. 27-35.

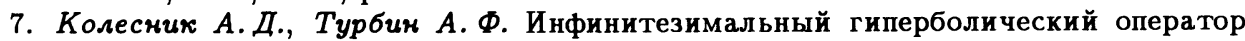
марковских случайных эволюций в $R^{n}$. - Докл. АН Украины, 1991, т. 1, с. 1114. 
8. Kolesnik A.D., Turbin A.F. The equation of symmetric Markovian random evolution in a plane. - Stochastic Process. Appl., 1998, v. 75, p. 67-87.

9. Orsingher $E$. Probability law, flow function, maximum distribution of wave-governed random motions and their connections with Kirchoff's laws. - Stochastic Process. Appl., 1990, v. 34, p. 49-66.

10. Orsingher $E$. Exact joint distribution in a model of planar random motion. - Stochastics Stochastics Rep., 2000, v. 69, № 1, p. 1-10.

11. Orsingher $E$., Bassan $B$. On a $2 n$-valued telegraph signal and the related integrated process. - Stochastics Stochastics Rep., 1992, v. 38, p. 159-173.

12. Орсингер Э., Колесних А.Д. Точное распределение в модели случайного движения на плоскости, управляемого гиперболическим уравнением четвертого порядка. - Теория вероятн. и ее примен., 1996, т. 41, в. 2, с. 451-459.

13. Orsingher E., San Martini A. Planar random evolution with three directions. Exploring Stochastic Laws, Ed. by A.V. Skorokhod. Utrecht: VSP, 1995, p. 357-366.

14. Ратанов $H$. E. Случайные блуждания частицы в неоднородной одномерной среде с отражением и поглощением. - Теорет. матем. физика, 1997, т. 112, № 1 , c. 81-91.

\section{СТОХАСТИЧЕСКИЕ ТРАНСПОРТНЫЕ СЕТИ И УСТОЙЧИВОСТЬ ДИНАМИЧЕСКИХ СИСТЕМ ${ }^{1)}$}

Рассмотрена сеть, содержащая $N$ узлов и $r N$ приборов, первоначально находящихся в узлах. В каждый узел поступает пуассоновский поток заявок интенсивности $\lambda(t)$. Заявка, попавшая в пустой узел, покидает систему. Если в узле есть приборы, то из них равновероятно выбирается прибор, который забирает заявку и перемещает ее в случайный узел, который выбирается равновероятно. Время перемещения распределено экспоненциально со средним значением 1 . Число обслуживающих приборов в каждом из $N$ узлов не превосходит $m$.

Мы исследуем устойчивость предельного детерминированного процесса, получаемого при $N \rightarrow \infty$. Далее, мы применяем наши результаты к системе массового обслуживания со сложной дисциплиной выбора прибора.

Ключевые слова и фразы: марковские процессы, нелинейные динамические системы, глобальная асимптотическая устойчивость, производящий оператор, сходимость, метод среднего поля, теория массового обслуживания.

\section{1. Введение}

В реалистических моделях стохастических транспортных сетей практически невозможно получить явные формулы, описывающие поведение системы. Асимптотический подход (при большом числе приборов) применялся в ряде последних исследований на эту тему (см. [1], [4], [6]). Мы получаем достаточные признаки глобальной асимптотической устойчивости и применяем их $\mathrm{x}$ двум системам, возникшим из современной теории массового обслуживания.

* Московский государственный университет им. М.В. Ломоносова, механикоматематический факультет, Воробьевы горы, 119899 Москва, Россия; e-mail: oseled@mech.math.msu.su, dima@vvv.srcc.msu.su

1) Работа первого автора поддержана РФФИ (гранты № 960100377 и № 99-0101104). Работа второго автора частично поддержана фондом ISSEP (грант s98-2042). 